\title{
Review of Non-Linear Transform based Techniques to Reduce the PAPR in OFDM Systems
}

\author{
Prabhjot Singh \\ ME Student \\ PEC University Of technology \\ Chandigarh(INDIA)
}

\author{
Divya \\ Associate Professor \\ PEC University Of technology \\ Chandigarh(INDIA)
}

\begin{abstract}
Orthogonal Frequency Division Multiplexing (OFDM) is a spectrum efficient multicarrier modulation scheme for high speed communication systems. OFDM provides robustness against multipath fading channels, but is sensitive to nonlinear effects due to the high Peak-to-Average Power Ratio (PAPR) of the transmitted signal. The reduction in PAPR is desirable in order to obtain power efficiency and to increase BER performance. This paper explores efficient Non Linear Transformation (NLT) algorithms for optimizing the PAPR of the OFDM signals. The proposed techniques avoid the use of additional Inverse Fast-Fourier Transform (IFFT) as compared to Selective Level Mapping (SLM) and Partial Transmit Sequence (PTS) and hence reduce the computational complexity. NLT schemes do not require the transmission of Side Information (SI) to the receiver, which provides better bandwidth utilization
\end{abstract}

\section{Keywords}

OFDM, PAPR, CCDF, SLM, PTS, Non Linear Transformation, L2-By-3.

\section{INTRODUCTION}

OFDM is a spectrum efficient multicarrier modulation method that converts a high data rate stream into a number of lower data rate streams, which are transmitted simultaneously over a number of subcarriers which are orthogonal to each other. The OFDM technique divides the frequency selective channel into a number of flat narrow band channels. These narrow band channels are overlapping and orthogonal to each other. Each sub channel is modulated with a separate symbol and then the $\mathrm{N}$ sub channels are frequency multiplexed. A block diagram of the OFDM system in a baseband model is shown in Fig 1.

In the transmitter, the binary inputs are first grouped to obtain a symbol in a baseband modulation, such as quadrature phase shift keying (QPSK) or M-ary quadrature amplitude modulation (MQAM) with $\mathrm{M}=2, \mathrm{M}=4, \mathrm{M}=16$, and so on. Then the serial input symbols are converted into a parallel vector form, which can be denoted as follows: $X=[X(0), X$ $(1), \ldots, \mathrm{X}(\mathrm{N}-1)$ ]. Each subcarrier is modulated by the QAM symbols in the data vector using the Inverse Fast Fourier Transform.

The OFDM signal can be expressed as

$x(n)=\sum_{k=0}^{N-1} X(k) e^{-j 2 \pi \frac{n}{N} k}$ for $0 \leq \mathrm{n} \leq \mathrm{N}-1$

Where, $x(k)$ is transmitted information symbol for the $\mathrm{k}^{\text {th }}$ subcarrier in the OFDM symbol. At the receiver FFT is used to recover data, which can be expressed as

$X(m)=\frac{1}{\sqrt{N}} \sum_{k=0}^{N-1} x(n) e^{j 2 \pi \frac{n}{N} m}$

equations (1) and (2) give a picture of the advantages of OFDM that it transfers the complexity of transmitters and receivers from the analogue to the digital domain making use of IFFT operation at the transmitting side and the FFT operation at the receiving end [1].

The inter symbol interference (ISI) can be eliminated to a large extent by using the cyclic prefix (CP). A guard time is introduced for each OFDM symbol after the IFFT, where the OFDM symbol is cyclically extended, as shown in 2 . This cyclical extension is called the cyclic prefix $(\mathrm{CP})$ [1].

Despite having advantages like bandwidth efficiency, simplification of channel equalization, robustness to multipath fading, and low computational complexity based on using the fast Fourier transform (FFT) technique, the major drawback of OFDM is its high peak- to-average power ratio (PAPR). High PAPR degrades performance of OFDM signals by forcing the analog amplifiers and D/A converters to work in nonlinear region. This results in distortion of the signal and high BER ratio. 


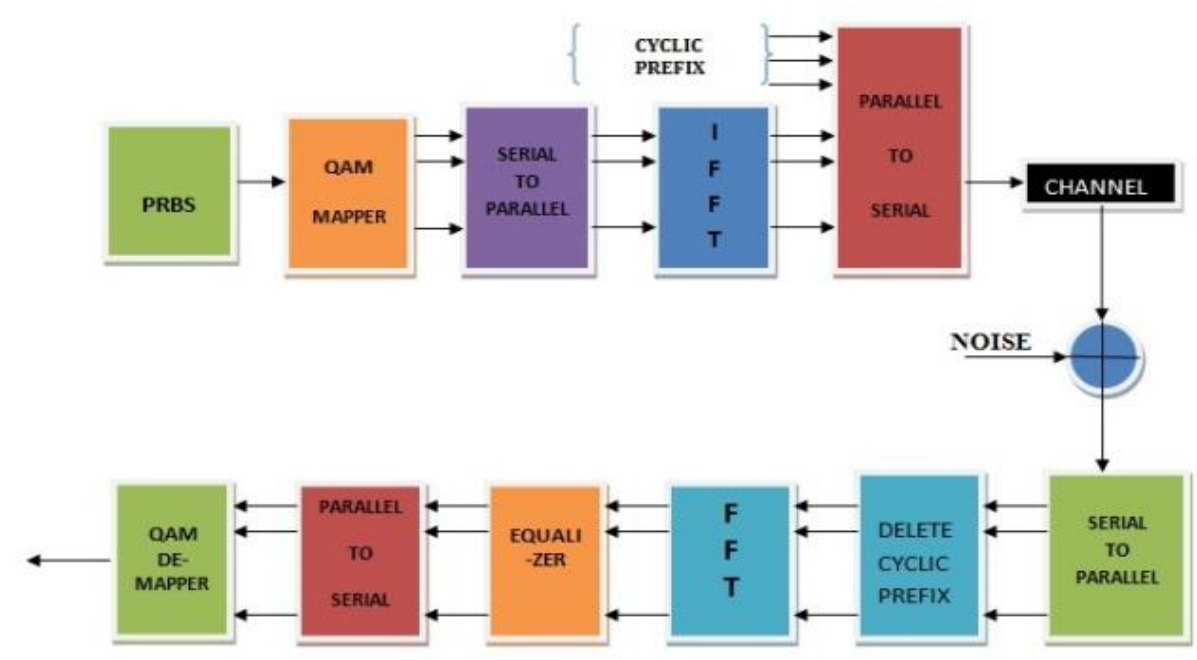

Fig 1: OFDM System

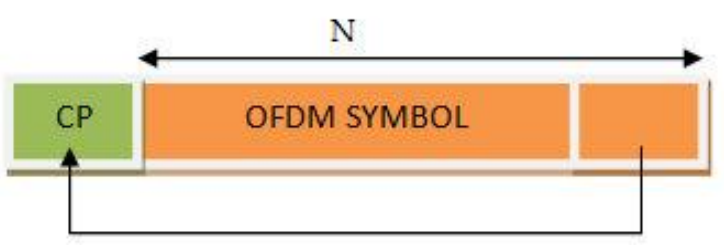

Fig 2: Cyclic Prefix addition

\section{Peak- To-Average Power Ratio}

The PAPR of continuous time signal is given by

$$
P A P R=\frac{\max |S(t)|}{|\overline{S(t)}|^{2}}
$$

Where $|\overline{S(t)}|^{2}$ denotes the transmitted signal mean power. To evaluate the PAPR performance the CCDF (complimentary commulative distribution function) of the PAPR is used. The $\mathrm{CCDF}$ function can be written as follows:

$\mathrm{CCDF}=\operatorname{Pr}(\mathrm{PAPR} \geq \mathrm{PAPR} 0)$

Fig 3 shows the PAPR's CCDF distribution with different number of sub-carriers (i.e. $N=64, N=128, N=256, N=$ 1024). The $x$-axis represents the PAPR thresholds while the $y-$ axis represents the probability of CCDF. As can be seen from the graph, for a given PAPR threshold, the appearance probability of OFDM symbols which above this threshold PAPR0 will decrease with the increase of sub-carriers number $N$.

Various methods have been proposed to solve the PAPR reduction problem in OFDM systems. Some of the important PAPR reduction methods are: amplitude clipping and filtering, partial transmit sequence (PTS), selected mapping (SLM), coding, interleaving, tone reservation (TR), tone injection (TI), and active constellation extension (ACE) [2].

Fig 4 shows the PAPR's CCDF distribution with SLM and PTS PAPR reduction techniques[3]. Form fig it is clear that there is $2.5 \mathrm{~dB}$ and $4 \mathrm{~dB}$ reduction in PAPR with SLM and PTS respectively.

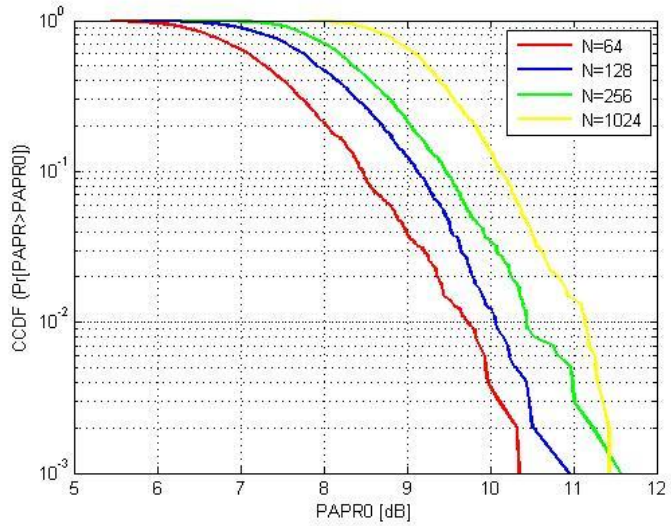

Fig 3: PAPR's CCDF curve of OFDM signal for different valves of $N$.



Fig 4: PAPR reduction performances of PTS algorithm and SLM algorithm.

At the expense of PAPR reduction, these methods results in the loss of data rate and increase in computational complexity. For example, SLM and PTS require side information to be sent to receiver[4]-[7], which results in the loss in data rate and extra power consumption. TR, TI, and ACE require a power increase in the transmit signal after PAPR reduction employed. The complexity is increased at both transmitter and receiver for these methods. 


\section{Non-Linear Transformation}

To overcome the shortcomings of these traditional PAPR reduction techniques several nonlinear mapping algorithms has been developed. These are [8][9][10]. Fig 5 shows the scheme implementation. $x_{n}=\sqrt{x_{n}^{2}} \operatorname{sgn}\left(y_{n}\right)$

Where "sgn(.)" is the sign function.

\section{(b) Modified Sliding Norm Transform (MSNT)}

The modified sliding norm transform (MSNT), which is a new

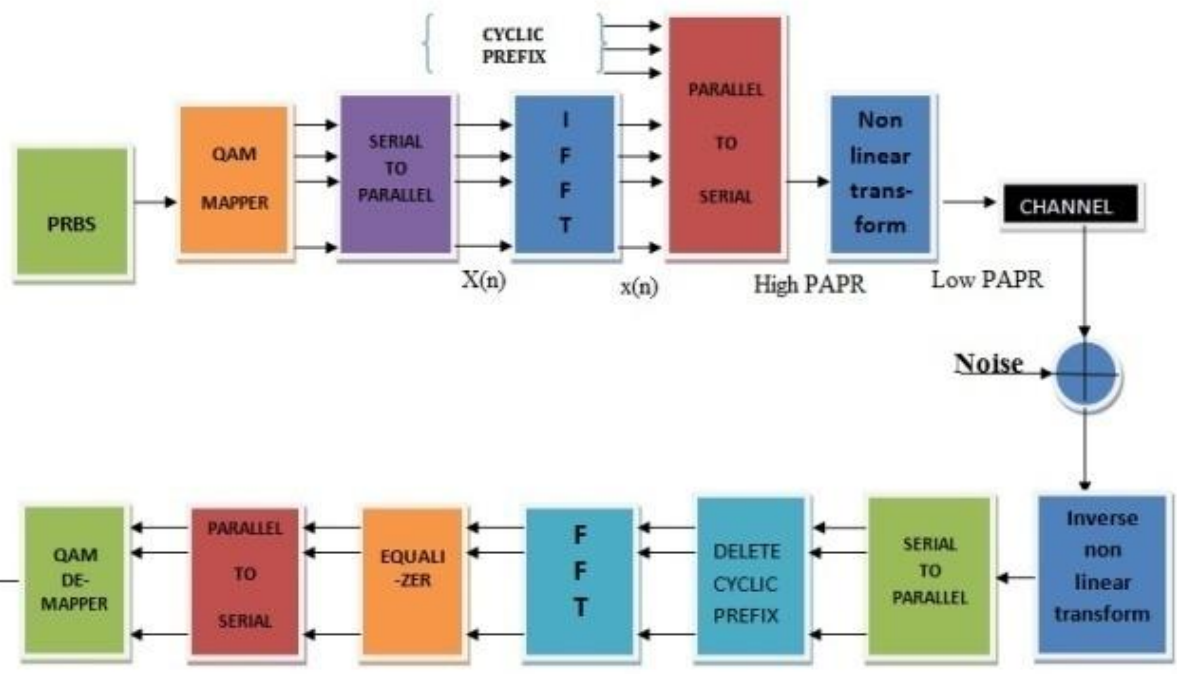

Fig 5: OFDM System with NLT PAPR reduction scheme.

\section{(a) Dursun method (L2-by-3)}

In this section, L2-by-3, nonlinear algorithm first proposed by Dursun is reviewed. First, the general definition of DSNT is introduced. Let $\mathrm{x}$ be a real vector with $\mathrm{N}$ samples denoted by $\mathrm{x}=[\mathrm{x}(0) \mathrm{x}(1) \ldots \mathrm{x}(\mathrm{N}-1)]$. A nonlinear discrete transform, as shown in (5) converts real vector value " $x$ " into " $y$," another real vector value.

$y_{n}= \begin{cases} & x_{0}, \quad n=0 \\ \frac{x_{n}}{\sqrt{\sum_{k=0}^{n} x_{k}^{2}}} \quad n=1,2, \ldots . . N-1\end{cases}$

The above transform is called the discrete L2-sliding norm transform (L2-DSNT). The L2-by-3 transform is a particular kind of DSNT method based on the L2 metric. It uses three samples, $\mathrm{x}_{\mathrm{n}-1}, \mathrm{x}_{\mathrm{n}}, \mathrm{x}_{\mathrm{n}+1}$, in each sliding window to calculate the output samples. The following equation shows the L2-by-3 mapping algorithm[8].

$y_{n}=\frac{x_{n}}{\sqrt{\alpha+x_{n-1}{ }^{2}+x_{n}{ }^{2}+x_{n+1}{ }^{2}}}$

This transform is reversible, and anyone can obtain the nonzero original data through.

$$
\begin{aligned}
& {\left[\begin{array}{cccccc}
\Delta_{0} & 1 & 0 & 0 & 0 & 1 \\
1 & \Delta_{1} & 1 & 0 & 0 & 0 \\
0 & 1 & \Delta_{2} & 1 & 0 & 0 \\
- & - & - & - & - & - \\
0 & 0 & - & 1 & \Delta_{N-2} & 1 \\
1 & 0 & 0 & 0 & 1 & \Delta_{N-1}
\end{array}\right]\left[\begin{array}{c}
x_{0}{ }^{2} \\
x_{1}{ }^{2} \\
x_{2}{ }^{2} \\
x_{N-2}{ }^{2} \\
x_{N-1}{ }^{2}
\end{array}\right]=-\alpha\left[\begin{array}{l}
1 \\
1 \\
1 \\
1 \\
1 \\
1
\end{array}\right]} \\
& \Delta_{n}=1-\frac{1}{y_{n}^{2}}
\end{aligned}
$$

The result obtained using (7) is the squared value of any nonzero data as denoted by $x_{n}^{2}$ and must be converted to the original data as $\mathrm{x}_{\mathrm{n}}$ using (8). nonlinear algorithm based on the L2-by-3 method with some modifications is proposed in [9]. The proposed nonlinear mapping function is defined as follows:

$y_{n}=\left\{\begin{array}{c}x_{0}, \quad n=0 \\ \frac{x_{n}}{\sqrt{a+b .\left|x_{n}\right|^{2}+\left|x_{n+1}\right|^{2}}} n=1,2 \ldots N-1\end{array}\right.$

The proposed algorithm is reversible, so at the receiver side following function can be applied to get the corresponding input data stream.

$x_{n}=\left\{\begin{array}{c}y_{0}, \quad y_{n} \neq 0, n=0 \\ y_{n} \cdot \sqrt{\frac{a+\left|x_{n-1}\right|^{2}}{1-b \cdot\left|y_{n}\right|^{2}}} \\ 0 \quad y_{n}=0\end{array}\right.$

\section{(c) Reduced Complexity Max Norm (RCMN)} algorithm

RCMN is reviewed here. The input data streams are mapped onto Quadrature Amplitude Modulation (QAM) constellation, then applied to IFFT. RCMN transformation is then performed on the time domain signal. The inverse transformation proposed at the receiver effectively recovers the original data blocks. The RCMN algorithm steps at the transmitter are detailed as follows[10]:

1. Generate the input data $\mathrm{x}=\left(\mathrm{x}_{1}, \mathrm{x}_{1}, \ldots, \mathrm{x}_{\mathrm{n}}\right)$ and map with the BPSK or QAM constellation and get the modulated data stream $X$.

2. Calculate IFFT for the mapped data stream $\mathrm{x}=\operatorname{IFFT}(\mathrm{X})$.

3. Find the maximum value from the IFFT output.

4. $\|x\| \max =\max (x 1, x 2, \ldots, x i, \ldots, x N)\|x\| \max =x i$ 
5. Define the parametric form of maximum norm by introducing the parameter $\alpha$. Multiply $\|x\| \max$ with the value of $\alpha$.

$\alpha^{*}\|\mathrm{x}\| \max =\alpha^{*} \max \left(\mathrm{x}_{1}, \mathrm{x}_{2}, \ldots, \mathrm{x}_{\mathrm{i}}, \ldots, \mathrm{x}_{\mathrm{n}}\right) \alpha^{*}\|\mathrm{x}\| \max =\alpha^{*} \mathrm{xi}$

where ' $\alpha$ ' is a parameter, that adjusts the PAPR of the transformed output. Optimized value of $\alpha$ ranges from 2 to 5 .

6. The output is transformed using RCMN technique

$$
\begin{array}{ll}
\text { a. } & y=x-\alpha^{*} x i \ldots \ldots \ldots \ldots \ldots \ldots \ldots,(\mathbf{A}) \\
\text { b. } & y=\left(\left(x_{1}-\alpha^{*} x_{i}\right),\left(x_{2}-\alpha^{*} x_{i}\right), .,\left(x_{i}-\alpha^{*} x_{i}\right), . .,\right. \\
& \left.\left(x_{N^{-}} \alpha^{*} x_{i}\right)\right) \\
\text { c. } \quad & y=\left(\left(x_{1}-\alpha^{*} x_{i}\right),\left(x_{2}-\alpha^{*} x_{i}\right), \ldots,(1-\alpha)^{*} x_{i}, \ldots,\right. \\
& \left.\left(x_{N^{-}} \alpha^{*} x_{i}\right)\right)
\end{array}
$$

7. Transmit the transformed output which offers low PAPR.

The RCMN algorithm steps at the receiver are as follows:

1. Receive the transmitted data block y.

2. Find the minimum value of $y$,

$\min (\mathrm{y})=\min \left(\left(\mathrm{x}_{1^{-}} \alpha^{*} \mathrm{x}_{\mathrm{i}}\right),\left(\mathrm{x}_{2}-\alpha^{*} \mathrm{x}_{\mathrm{i}}\right), \ldots,(1-\alpha)^{*} \mathrm{x}_{\mathrm{i}}, \ldots,\left(\mathrm{x}_{\mathrm{N}^{-}}\right.\right.$ $\left.\alpha^{*} \mathrm{x}_{\mathrm{i}}\right)$ )

$\min (\mathrm{y})=(1-\alpha) * \mathrm{x}_{\mathrm{i}}$

3. Divide $\min (\mathrm{y})$ by $(1-\alpha)$ to obtain $\mathrm{x}_{\mathrm{i}} \min (\mathrm{y}) /(1-\alpha)=$ $\mathrm{x}_{\mathrm{i}}$

4. To obtain $\mathrm{x}$, add $\alpha^{*} \mathrm{x}_{\mathrm{i}}$ with $\mathrm{y}$. From equation (A)

$\mathrm{x}=\mathrm{y}+\alpha^{*} \mathrm{xi}$

\section{SIMULATIONS RESULTS}

PAPR analysis in OFDM Systems is done by comparing the PAPR for OFDM transmitted signal after applying the Non linear Transform (L-2 by 3, MSNT, RCMN) and simple OFDM transmitted signal. Another evaluation done in this paper is the complexity analysis.

Fig 6 shows the PAPR reduction performance of L2-by-3 transform at $(\mathrm{a}=0.2,0.4,0.6,0.8)$. The method provides good reduction at a 0.2 and 0.1 however at $\mathrm{a}=0.2$ is the one at which required $\operatorname{BER}\left(1 \times 10^{-4}\right)$ is obtained[8].

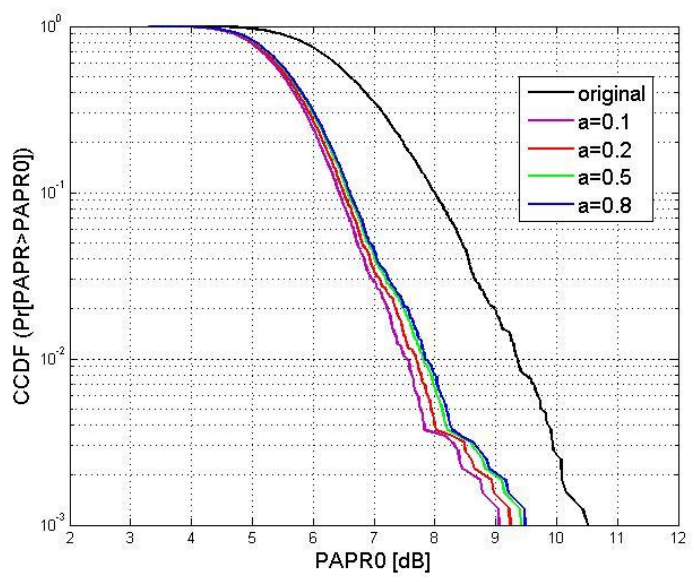

Fig 6: PAPR reduction performance for $L 2-b y-3$ for $a=$ $[0.2,0.4,0.6,0.8]$

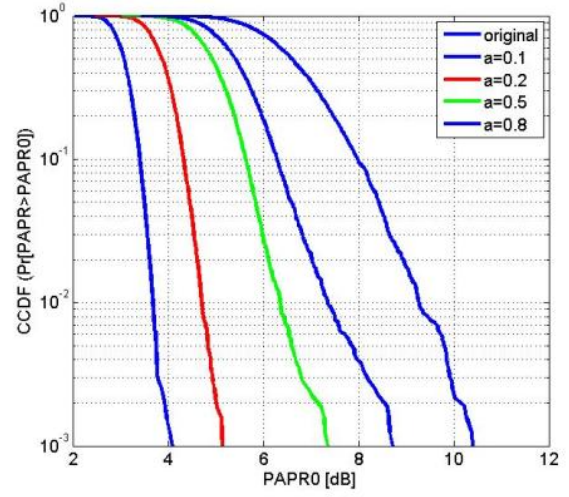

Fig 7: PAPR reduction performance for MSNT when $\mathrm{a}=$ $[0.1,0.2,0.5,0.8]$

Fig 7 shows the PAPR reduction performance of MSNT with different values of ' $a$ ' and where ' $b$ ' $=8$, and the baseband modulation is 4 QAM and $N=64$. It is shown that increasing ' $a$ ' from 0.1 to 1 the PAPR of the output signal varies from 4 $\mathrm{dB}$ to $8.5 \mathrm{~dB}$

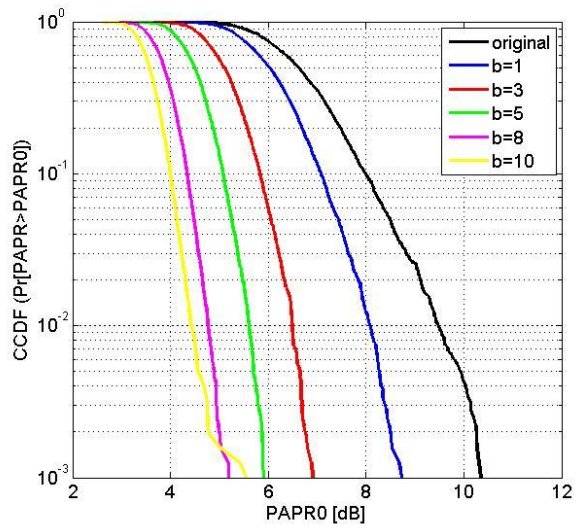

Fig 8: PAPR reduction performance for MSNT when $b=$ $[1,3,5,8,10]$ with $\mathrm{a}=0.2$

In the next step, the performance of MSNT with variations of $\mathrm{b}$ in the range 1 to 10 has been investigated. Fig 8 shows that as the $b$ increases the PAPR decreases significantly. As shown in the figures, the PAPR is reduced from $8.5 \mathrm{~dB}$ to $5 \mathrm{~dB}$. MSNT results in better performance than using the conventional OFDM technique, and there is no sensitivity to $b$ variations in the PSD, as in [9]

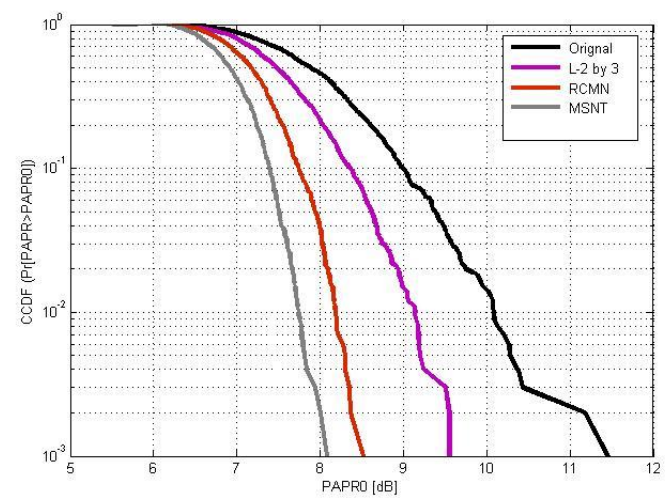

Fig 9: CCDF comparison of L2-by-3, RCMN \& MSNT. 
Fig 9 shows PAPR reduction performance of L2-by-3, RCMN \& MSNT. It should be noted that the comparison of these methods is based on the PAPR reduction amount at the probability of clipping level of $1 \times 10^{-3}$. L2-by-3 at a $=0.2$, MSNT with $a=0.2, b=8$ RCMN with $a=2$.

\section{Complexity Analysis}

Another evaluation of NLT methods is complexity analysis. Complexity of the mentioned algorithms can be computed with respect to the number of additions and multiplications of real numbers. For optimum results MSNT sub-block is used twice; therefore, $10(\mathrm{~N}-1)$ real multiplications and $8(\mathrm{~N}-1)$ real additions is needed in any OFDM symbols that include $\mathrm{N}$ samples. L2-by3 transform needs 6(N-1) additions and 6(N-1) multiplications. MSNT algorithm has high complexity with respect to the L2-by-3 algorithm. Complexity of RCNT is least, as it involves only $\mathrm{N}$ additions and $\mathrm{N}$ multiplications. Number of additions and multiplications of PAPR reduction algorithms are listed in Table 1.

\begin{tabular}{|c|c|c|}
\hline Methods & $\begin{array}{l}\text { Additional } \\
\text { complexity }\end{array}$ & $\begin{array}{l}\text { Multiplication } \\
\text { complexity }\end{array}$ \\
\hline SLM & $\begin{array}{l}\operatorname{DNL}(3 \log (\mathrm{N})+2) \\
+2 \mathrm{~N}(\mathrm{D}-1)\end{array}$ & $\begin{array}{l}2 \mathrm{DNL}(\log (\mathrm{N})+2) \\
+4 \mathrm{~N}(\mathrm{D}-1)\end{array}$ \\
\hline PTS & $\begin{array}{l}\text { 4(D-1/2)NLV- } \\
\text { DNL+V*A } A_{\text {IFFT }}\end{array}$ & $\begin{array}{l}4(\mathrm{D}-1) \mathrm{NLV}- \\
2 \mathrm{DNL}+\mathrm{V}^{*} \mathrm{M}_{\mathrm{IFFT}}\end{array}$ \\
\hline L2-by-3 & $6(\mathrm{~N}-1) \mathrm{L}$ & $6(\mathrm{~N}-1) \mathrm{L}$ \\
\hline MSNT & $8(\mathrm{~N}-1) \mathrm{L}$ & $10(\mathrm{~N}-1) \mathrm{L}$ \\
\hline RCNT & $\mathrm{NL}$ & $\mathrm{NL}$ \\
\hline
\end{tabular}

Table 1: Complexity of NLT algorithm and traditional SLM and PTS algorithms.(D-number of subblocks in SLM, V-number of subblocks in PTS, L-oversampling factor)

\section{CONCLUSION}

Non-Linear Transform PAPR reduction techniques in OFDM systems are explored. It is shown that the NLT methods reduce PAPR of the OFDM signal and thus increases the efficiency of power amplifier, increases system performance by reducing in-band and out-of-band distortions. These NLT transforms are applicable independently of the signal constellation and mapping on subcarriers, and reduces PAPR at low computational complexity. These transformations can provide more PAPR reduction than that of SLM \& PTS by only changing a value, and this value can be used by transmitter and receiver as a convention; it does not have to be transmitted as required in SLM \& PTS.

\section{REFERENCES}

[1] Y. Wu and W. Y. Zou, "Orthogonal frequency division multiplexing: a multi-carrier modulation scheme," IEEE Trans. Consum. Electron., vol. 41, no. 3, pp. 392-399, 1995.

[2] T. Jiang and Y. Wu, "An Overview: Peak-to-Average Power Ratio Reduction Techniques for OFDM Signals," IEEE Trans. Broadcast., vol. 54, no. 2, pp. 257-268, Jun. 2008.

[3] W. Yi and G. Linfeng, "An Investigation of Peak-toAverage Power Reduction in MIMO-OFDM Systems," Masters Thesis,Blekinge Inst. Technol., no. October, 2009.

[4] M. Breiling, S. H. Müller-weinfurtner, and J. B. Huber, "SLM Peak-Power Reduction Without Explicit Side Information," IEEE Commun. Lett., vol. 5, no. 6, pp. 239-241, 2001.

[5] C. Pradabpet, Y. Yoshikazu, M. Sorawat, and C. Kobchai, "New PTS Method with Coded Side Information Technique for PAPR Reduction in OFDM Systems," in International Symposium on Communications and Information Technologies (ISCIT 2008), 2008, vol. 45888, no. 1, pp. 104-109.

[6] V. . Malode and B. P. Patil, "PAPR Reduction Using Modified Selective Mapping Technique," Int. J. Adv. Netw. Appl., vol. 02, no. 02, pp. 626-630, 2010.

[7] S.-J. Heo, H.-S. Noh, J.-S. No, and D.-J. Shin, "A Modified SLM Scheme with Low Complexity for PAPR Reduction of OFDM Systems," 2007 IEEE 18th Int. Symp. Pers. Indoor Mob. Radio Commun., pp. 1-5, 2007.

[8] S. Dursun and A. M. Grigoryan, "Nonlinear L2-by-3 transform for PAPR reduction in OFDM systems," Comput. Electr. Eng., vol. 36, no. 6, pp. 1055-1065, Nov. 2010.

[9] R. Salmanzadeh and B. M. Tazehkand, "A Modified Method Based on the Discrete Sliding Norm Transform to Reduce the PAPR in OFDM Systems," ETRI J., vol. 36, no. 1, pp. 42-50, Feb. 2014.

[10] M. Palanivelan and S. Anand, "Reduced Complexity Max Norm based PAPR Optimization in OFDM systems," WSEAS Trans. Commun., vol. 11, no. 5, pp. 171-181, 2012. 